\title{
A Survey of Therapeutic Drug Monitoring status in China
}

Liang Yan ${ }^{1}$, Huang Qi ${ }^{2}$, Bo-Ting Zhou ${ }^{3}$, Tao Yin ${ }^{2}$, Mimi Tang ${ }^{1}$, Jiang lou $^{2}$, and Xiang Dong $^{2}$

${ }^{1}$ Xiangya Hospital Central South University

${ }^{2}$ Affiliation not available

${ }^{3}$ Xiangya Hospital, Central South University

June 20, 2021

\section{Abstract}

In order to comprehensively understand the current situation of therapeutic drug monitoring in China, a questionnaire survey was conducted among doctors, pharmacists and laboratory doctors in hospitals at all levels by using Internet technology and mobile phone client.

\section{Title:A Survey ofTherapeutic Drug Monitoring status in China}

Hongyan Liang ${ }^{1,2,3, \#}$, Qi Huang ${ }^{1,2,3, \#}$, Mimi Tang ${ }^{1,2,3}$, Jiang Lou $^{4}$, Dong Xiang ${ }^{5}$, Tao Yin ${ }^{1,2,3, *}$, Boting $\mathrm{Zhou}^{1,2,3, *}$

1. Department of Pharmacy,Xiangya Hospital, Central South University,Changsha 410008,China

2. The Hunan Institute of Pharmacy Practice and Clinical Research, Changsha 410008,China

3. Institute of Hospital Pharmacy,Central South University,Changsha 410008, China

4. Affiliated Hangzhou First People's Hospital, Zhejiang University School of Medicine, Hangzhou 310003, China

5.Department of Pharmacy, Tongji Hospital Affiliated with Tongji Medical College, Huazhong University of Science and Technology, Wuhan 430030, China

\#Equal contributors

*Corresponding authors:

Tao Yin, simon863@vip.sina.com. Department of Pharmacy,Xiangya Hospital, Central South University,Changsha 410008,China

Boting Zhou, botingzhou0918@126.com. Department of Pharmacy,Xiangya Hospital, Central South University,Changsha 410008,China

\section{Hosted file}

A Survey of Therapeutic Drug Monitoring status in China.doc available at https://authorea. com/users/420753/articles/527017-a-survey-of-therapeutic-drug-monitoring-status-in-china 


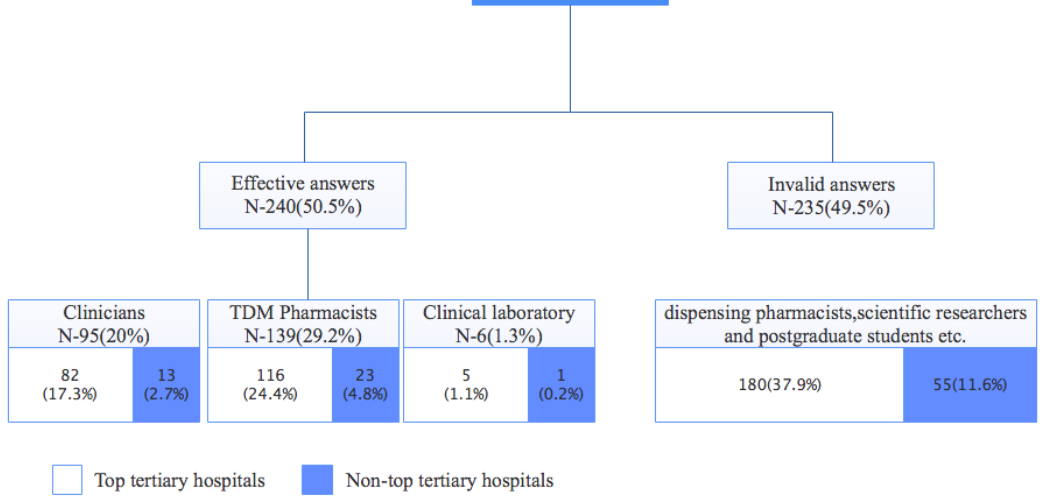

Fig 1. The occupational distribution of participants. Effective rate (50.5\%) refers to the ratio of the total number of clinicians, TDM pharmacists and clinical laboratory physicians to the total number of respondents. 


\section{Hosted file}

Fig2 .docx available at https://authorea.com/users/420753/articles/527017-a-survey-oftherapeutic-drug-monitoring-status-in-china 